\section{Controlling adolescent B cells}

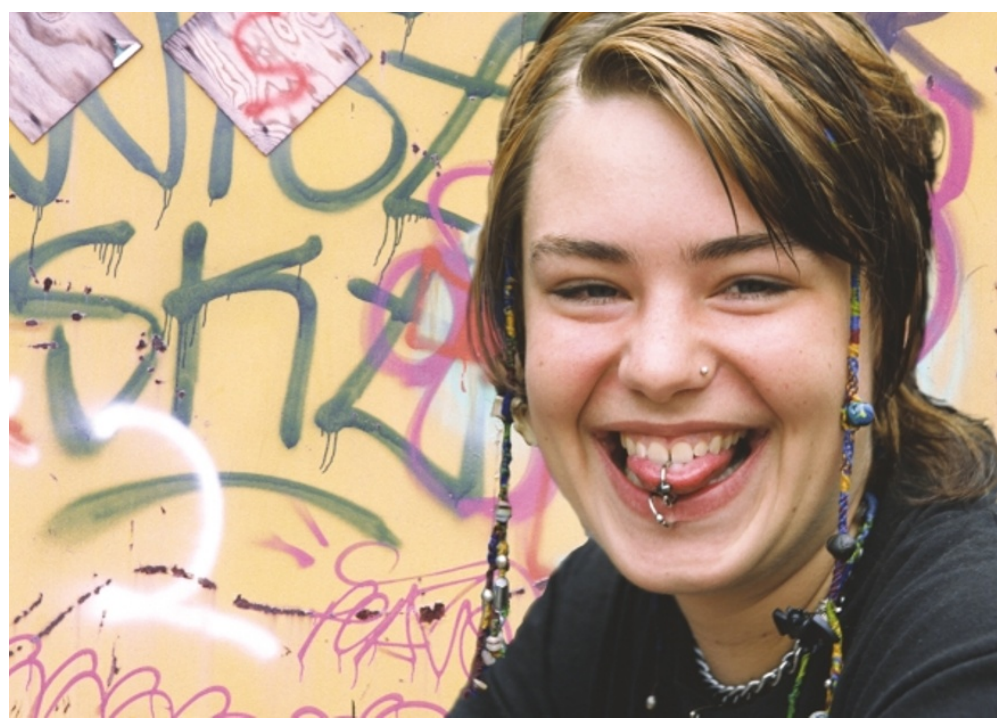

Less than $10 \%$ of the immature B cells that exit the bone marrow will complete their development in the spleen to become mature follicular or marginal-zone B cells. However, little is known about the molecular mechanisms that govern this final rite of passage. Now, a report in The Journal of Immunology indicates that Id 2 has an important role.

Transcription factors that contain a basic helix-loop-helix (bHLH) motif have been shown to be crucial for various cell-differentiation processes. The transcriptional activity of the bHLH proteins can be regulated by members of the Id family, another class of HLH proteins. Id proteins lack a DNA-binding region. Instead, they function solely by dimerization with the bHLH family, and they inhibit the functions of this family in a dominant-negative manner.

To examine the role of Id proteins in B-cell development in the spleen, Becker-Herman, Lantner and Shachar first measured the level of expression of Id proteins in immature and mature splenic B cells. Similar levels of Id 1 and Id 3 messenger RNA were detected in immature and mature B-cell populations; however, the expression of Id2 was markedly reduced in mature $\mathrm{B}$ cells.

The authors hypothesized that Id2 might limit B-cell maturation in the spleen. In support of this, Id2deficient mice were shown to have an increased number of mature splenic $B$ cells. Further analysis indicated that the accumulation of mature $B$ cells in $I d 2^{--}$mice was not owing to increased cellular life span or aberrant B-cell development in the bone marrow. Strikingly, although there is a greater number of mature B cells in $I d 2^{-1-}$ mice, marginal-zone $\left(\mathrm{IgM}^{+} \mathrm{IgD}^{-}\right.$ $\left.\mathrm{CD} 2 \mathrm{1}^{\mathrm{hi}}\right) \mathrm{B}$ cells are virtually absent.

A potential target of Id2 is the bHLH protein E2A, which is required for the initiation of B-cell development. Immature and mature B cells express equivalent levels of E2A mRNA. E2A-specific E2-box-binding
TOLERANCE

\section{T-cell turn-off}

A study that is published in Cell this month shows that NFAT (nuclear factor of activated $T$ cells) - a transcription factor that has a central role in full T-cell activation - can also initiate a genetic programme that leads to anergy.

Anergy is a state of lymphocyte nonresponsiveness that is induced by suboptimal antigen stimulation, and it is thought to be important in preventing harmful responses to self-antigens. But, the biochemical pathways that lead to anergy rather than full activation are poorly understood.

Macián and co-workers investigated whether the $\mathrm{Ca}^{2+}$ signalling pathway triggers a distinct genetic programme that leads to anergy. They used an in vitro model in which $\mathrm{CD} 4^{+} \mathrm{T}$ cells are treated with ionomycin - an activator of the $\mathrm{Ca}^{2+}$-calcineurin-NFAT signalling pathway - to mimic signalling through the T-cell receptor (TCR) only and induce anergy, or with PMA (phorbol 12-myristate 13-acetate) and ionomycin to mimic full activation by TCR signalling and costimulation (see figure). Microarray analysis of global gene expression showed reproducibly that the expression of $\sim 70$ genes was induced more strongly (or as strongly) by ionomycin alone than by PMA and ionomycin.

The ability of NFAT to regulate the expression of these anergy-associated genes was then assessed. NFAT1 is the

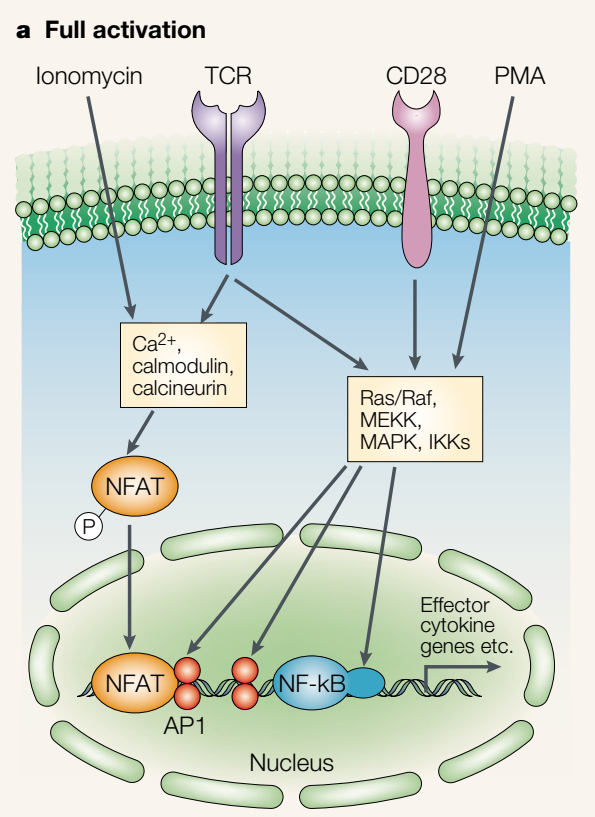

predominant member of the NFAT family in T cells, and in ionomycin-treated $\mathrm{Nfat}^{-/-}$ $\mathrm{T}$ cells, the expression of 15 out of 18 anergyassociated genes tested was reduced compared with wild-type T cells. $\mathrm{Nfat}^{-1-}$ $\mathrm{T}$ cells were less susceptible to the induction of anergy, which further indicates a role for NFAT in this process.

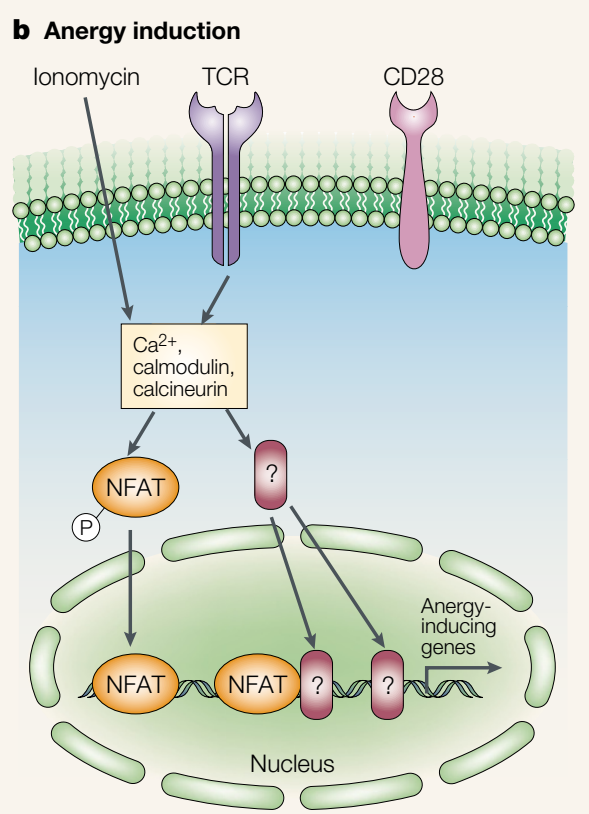


activity, however, was shown to be markedly reduced in immature $B$ cells. Immunoprecipitation studies showed directly that Id2 and E2A form heterodimers in immature $B$ cells, which indicates that Id 2 might be responsible for the inhibition of E2A function.

This study shows, for the first time, a role for Id 2 in the regulation of B-cell maturation in the spleen. In this process, it seems that Id 2 has two functions - it allows commitment to the marginal-zone B-cell lineage and controls the differentiation of follicular B cells. But, the genes that are regulated by $\mathrm{Id} 2$ at this stage of development have not been identified yet.

Jennifer Bell

(D) References and links ORIGINAL RESEARCH PAPER BeckerHerman, S., Lantner, F. \& Shachar, I. Id2 negatively regulates $\mathrm{B}$-cell differentiation in the spleen. J. Immunol. 168, 5507-5513 (2002) FURTHER READING Engel, I. \& Murre, C. The function of $\mathrm{E}$ - and ld proteins in lymphocyte development. Nature Rev. Immunol. 1, 193-199 (2001)

Interaction of NFAT with the nuclear factor AP1 is essential for the transcription of effector cytokine genes during T-cell activation, but is this interaction involved in the induction of anergy? Activation of AP1 was not detected in ionomycintreated T cells. Furthermore, the transduction of $\mathrm{T}$ cells with a constitutively active NFAT 1 that cannot interact with AP1 was sufficient to render the $\mathrm{T}$ cells anergic and to induce the expression of several anergy-associated genes. On the basis of their results, the authors propose a new model of T-cell anergy (see figure). Importantly, this model predicts that blockade of the NFAT-AP1 interaction should prevent $\mathrm{T}$-cell activation and induce a longlasting state of tolerance. This is a key goal of organ transplantation that current regimes of immunosuppression fail to achieve.

Jennifer Bell

(2) References and links ORIGINAL RESEARCH PAPER Macián, F. et al. Transcriptional mechanisms underlying lymphocyte tolerance. Cell 109, 719-731 (2002) WEB SITE

Anjana Rao's lab: http://www.hms.harvard.edu/ $\mathrm{dms} / \mathrm{bbs} / \mathrm{fac} / \mathrm{rao} . \mathrm{html}$

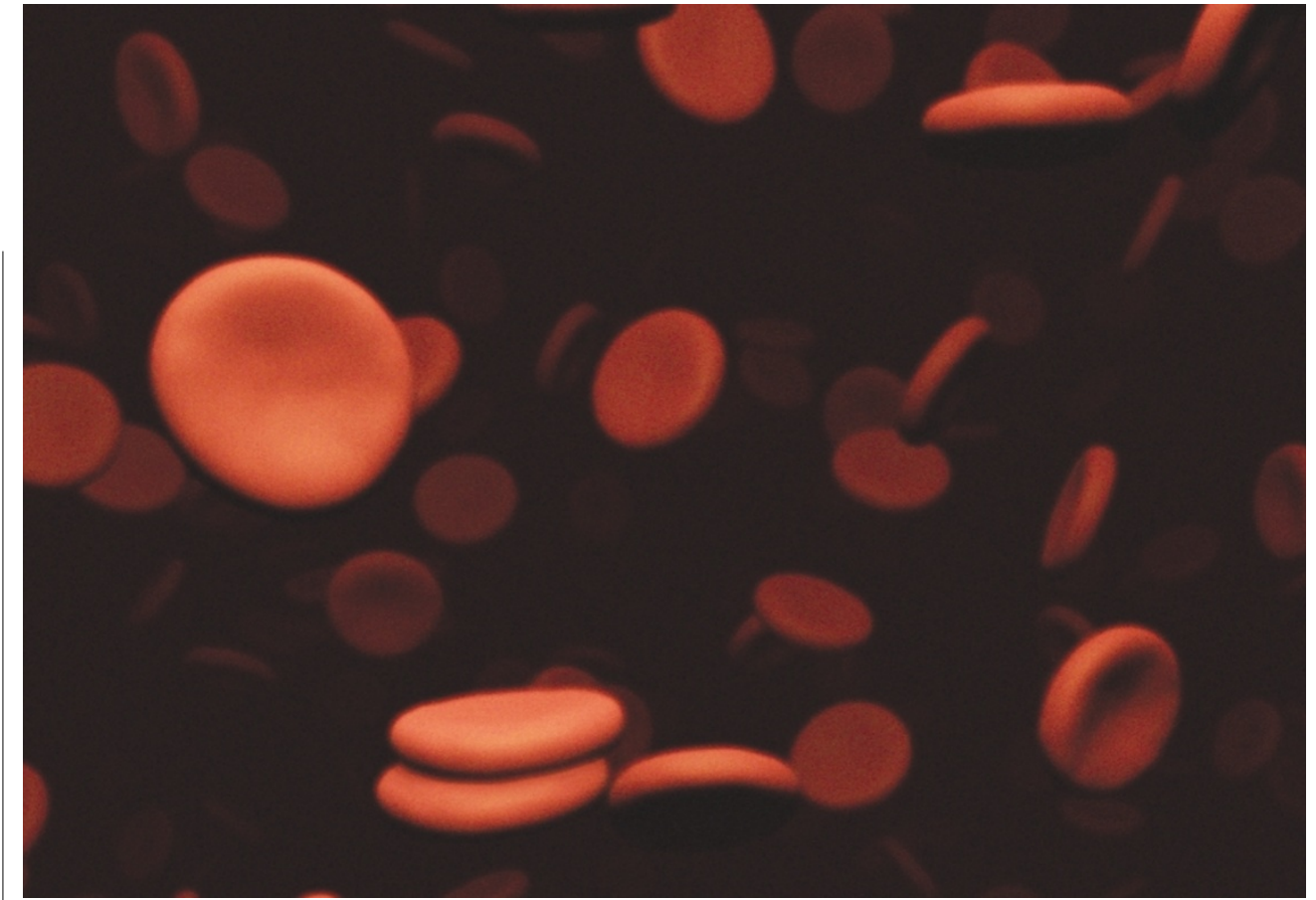

\section{HAEMATOPOIESIS}

\section{The origin of HSCs}

Despite intensive investigation, the origin of the first haematopoietic stem cells (HSCs) that develop during mammalian embryogenesis is unclear. Two recent studies published in Immunity provide clues as to the cellular origin of these HSCs and their location within the embryonic tissues from which they emerge.

HSCs give rise to all haematopoietic lineages and are also capable of self-renewal. In adult animals, HSCs reside in the bone marrow, but during mouse embryonic development, HSCs are found first in the dorsal aorta within the aorta-gonads-mesonephros (AGM) region and in the vitelline and umbilical arteries before the bone marrow forms.

The transcription factor Runx 1 and its heterodimeric protein partner core-binding factor- $\beta(\mathrm{CBF} \beta)$ are essential for the formation and/or function of HSCs, because the homozygous deletion of either of the genes that encode these proteins results in a total block in definitive haematopoiesis. In addition, Runx1 is expressed in all sites from which HSCs emerge. These previous studies had indicated that the expression of Runx1 marks the first HSCs in the embryo but, until now, this had not been shown directly.

North and colleagues used adoptive-transfer assays to determine whether Runx 1 is expressed in embryonic HSCs. They isolated Runx $1^{+}$and Run $\times 1^{-}$cells from the AGM region, vitelline and umbilical arteries, and livers of mouse embryos and then carried out transplantation assays. For each of the haematopoietic tissues tested, all HSCs were found in the Runx ${ }^{+}$population and no
HSCs were found in the Runx $1^{-}$population. Further experiments showed that most HSCs in wild-type embryos express endothelial cellsurface markers and CD45 (a leukocyte marker that is expressed by all haematopoietic cells except erythrocytes). By contrast, HSCs in Runx $1^{+/}$ embryos are heterogeneous, and include cells that express CD45, as well as $\mathrm{CD}^{-} 5^{-}$endothelial cells and mesenchymal cells. These studies, therefore, show that all HSCs in mouse embryos express Runx1, and that Runxl affects the distribution of HSCs into different cell populations in a dosedependent manner.

In the second study, De Bruijn et al. investigated the spatial localization of HSCs within the mouse embryo, using the Scal surface glycoprotein (a well-known marker of HSCs) and a green fluorescent protein (GFP) marker gene (expressed under the control of Ly-6A gene regulatory sequences, which encode Sca1). First, they established that the Ly-6A-GFP transgene is expressed by all functional HSCs in the AGM of embryonic day 11 mice; then, they carried out immunohistochemical staining to investigate where these cells reside. In the $\mathrm{AGM}, \mathrm{GFP}^{+}$cells were specifically localized to the single layer of $\mathrm{CD} 1^{+}$endothelial cells that lines the wall of the dorsal aorta, but were not found in the underlying mesenchyme. The authors concluded that HSCs are generated within the layer of endothelial cells that lines the wall of the dorsal aorta, and by additional flow-cytometric analysis, they showed that these cells have both endothelial and haematopoietic cell-surface characteristics. The authors hope that, on the basis of these findings, future studies might allow the immediate precursors of HSCs to be identified.

Jenny Buckland

(4) References and links

ORIGiNAL RESEARCH PAPERS North, T. E. et al. Runx 1 expression marks long-term repopulating hematopoietic stem cells in the midgestation mouse embryo. Immunity 16, 661-672 (2002) | De Bruijn, M. F. T. R. et al. Hematopoietic stem cells localize to the endothelial cell layer in the midgestation mouse aorta. Immunity 16, 673-683 (2002) 